\title{
System Properties to Address the Change Propagation in Product Realization
}

\author{
Mohammad ARJOMANDI RAD ${ }^{1}$, Roland STOLT and Fredrik ELGH \\ Department of Product Development, School of Engineering \\ Jönköping University, Jönköping, Sweden
}

\begin{abstract}
Demanding markets and complex products are only some of the reasons that make changes and variations inevitable through different stages of product realization. From early phases of product development to downstream production phase, these changes or variations cause failure either directly or by propagating to other phases, triggering more fluctuations like the well-known butterfly effect. In this paper, first, the definition of changes in product realization will be reviewed and then different papers and their classification on change related system properties (illites) will be discussed and compared. It was argued that considering a system-level view, one could trace these propagations in the systems as a result of not being robust, flexible, or adaptable, etc. Some of the ambiguity in this semantic field demonstrated and most repeated definitions are identified as the unanimous and agreed-upon definitions in the literature. In the end, a historical comparison of the three identified properties presented. The results of this study help us to understand the multidisciplinary nature of these propagations and identify their stemming turbulent environment. This will be used as a foundation for forthcoming research either to prevent these propagations or utilize their attributes in the product realization.
\end{abstract}

Keywords. Changes in product realization process, Change propagation, Chaotic behavior, Adaptable, Flexible, and Robust design, Product design and development, Mass customization

\section{Introduction}

Product realization is a transdisciplinary field of research that has one goal, and that is to fill out the requirements based on constraints or to be more precise, to address stakeholder's preferences [1]. The fundamental characteristic of these preferences is that they change. Regulations, innovative technologies, stakeholder's dynamic preferences, and complexity in product architecture lead to changes or a series of changes [2] sometimes addressed as propagation in the literature. A research project named Butterfly Effect has recently started in Jönköping School of Engineering to study and exploit these change propagations. The name comes from chaos theory by Edward Lorenz [3] that argues that unpredictable changes will prevent forecasting the occurrence of tornados in the future of our systems. This paper is the very first stage in this project aiming to clarify the research, find a suitable direction, and review the literature in a broader system view. Since the project has started newly, and no interviews have done with companies. This paper lies with the ontological and theoretical framework rather than industrial case

\footnotetext{
${ }^{1}$ Corresponding Author, Mail: mohammad.rad@ju.se.
} 
analysis. Therefore, the scope of this paper does not cover producing a chaos model and defining attractors, but such information can be a topic of future studies. Lorenz studied chaos in complex product family shape and its multiple composition factors [4].

Nonaka in his famous book [5] argued that changes in product and production design should be welcomed. Thus, changes and their propagations could have positive effects by creating opportunities to practice lean design or lean manufacturing and increase efficiency, or indeed they can have a negative effect and be a threat, triggering other changes, resulting in rework or longer lead time [6]. For example, Stolt et al. [7] talk about three companies with ETO business models that need supports for customization and easy adaptation to fluctuating requirements during the course of a development project. And [8] address this by acknowledging change as a normal condition and developing means to efficiently re-design and assess the impact of changes by adopting means like set-based concurrent engineering.

Propagation starts with a change. For example, making small changes in the product model could cause large and unexpected effects on the production system. Or vice versa, making changes in the production system may cause large and unpredicted effects in what can be offered to the customer through the product model [9]. To understand change propagation in product realization, the first logical step is to take a closer look at the change itself in a generic way and elaborate on what change means. Therefore, this paper will look at the broader picture with a system engineering [10] approach. System engineering is a transdisciplinary tool that focuses on the development of functionality in the early phases, based on customers' needs and requirements which continues into synthesis and validation [11]. Lifecycle properties or system properties, which sometimes are called ilities, aid designers with the right alternatives for stakeholders' preferences [12]. The transdisciplinary nature of this paper and the Butterfly Effect project is highlighted here when considering a wide range of disciplines involved, from product development to production and from system engineering to the computer science field.

The purpose of this paper is to find change-related system properties and a consensus definition for them in literature and identify the trend for future research in our research project. Therefore, this paper presents a review of change-related system properties and argues that Flexibility, Adaptability, and Robustness are the most used properties (ilities) that the majority of authors have agreed on them and use them for changeability in system level. Moreover, a historical comparison of the these most used ilities illustrates that recently Robustness is getting high recognition form scholars in this field.

\section{Literature review methodology}

Changes in product realization have been studied from many perspectives. Design for change is one such viewpoint that first proposed by [13] and entails several properties that together they offer the concept of changeability throughout the system lifecycle. Changeability in a complex system is an attribute that prevents change propagations [14] and preserves stakeholders' preferences. These properties are sometimes called ilities and have a very diverse range. Table 1 shows one basic classification for these properties.

Table 1. Change-Related and Architecture-Related ilities Examples [15].

\begin{tabular}{lll}
\hline \multicolumn{2}{c}{ Change-related ilities } & \multicolumn{2}{c}{ Architecture-related ilities } \\
\hline Adaptability, Flexibility, Reconfigurability, & Accessibility, Controllability, Modularity, \\
Agility, Changeability, evolvability, & Interoperability, Simplicity, Independence, \\
Extensibility, Modifiability, Scalability, & Interoperability, Integrability, Protectability, \\
Versatility, & & Readability, Decentralization \\
\hline
\end{tabular}


There are lots papers that study words and phrases that address these changes, fluctuations, or uncertainties especially about their scope and limitations. To identify change-related ilities this article used keywords on the left side of Table 1 to collect subsequent articles on this subject. For searching the articles Scopus and Google scholar are used together to maximize the results. No limitation was imposed on document type or time span in the searches. Excel was used to track the searches and archive them. Various combination of mentioned keywords has been used in a logical order. The whole work was iterative and new words and phrases were constantly added to the excel sheet to increase the depth of searches. As for our procedure, First, articles were filtered by their title and the results gave us the first round of articles to work with. Second, the selected articles' abstracts were read, and they were filtered based on relation to the topic by asking three major criterion questions used to limit the papers as below.

- Is it related to change related system properties (ilities)?

- Is it giving a definition for ilities or try to compare them (have a semantic view)?

- What domain does the paper belong to (product or production)?

Many articles (roughly $80 \%$ ) were found to be using the mentioned terms as the desired effect on their system. This means their focus was more on studying the method or mechanism and achieving these attributes without giving any definition on them. For example, a lot of articles employ tactics to get one ility as an attribute in their system. Yet for the purpose of the current paper, which is to hold on to the system engineering approach, these articles were not looked through.

Focusing on product development, some articles with the ontological view in production or manufacturing were also not included in the source papers. The reason for this is that changeability may have different semantic fields between product and production discipline and this difference falls out of the scope of this paper. However, for understanding the difference between the meaning of change-related ilities in product and production domains readers are directed to the article [16] and [17]. Nevertheless, some articles that have broader scope about systems in generic form and literature address them as system engineering papers. They are included in the search results considering that they satisfy other criterion questions.

\section{Recognizing system properties in change propagation}

As mentioned, in today's everchanging markets and dynamic environment, the key to success for companies is to be able to address late decisions or unanticipated problems and incorporate changes into their offerings even after its release into the market. Clark et al [18] stated that "Late implementation of a change from one phase to another phase (e.g., concept, manufacturing, operations) becomes 10 times more costly". They described this as the "Rule of Ten". Wildemann in 1994 was first to suggest three tactics to manage changes in the systems and Fricke et al completed it by adding two more tactics [13]. To name them, Prevention, Front loading, Effectiveness, Efficiency, and Learning. Later, [19] proposed the design for changeability. Their aim was to make a system more open to changes during its life cycle in dynamic markets. Figure 1 is the adoption of this concept. Flexibility is accomplished when the system can change 
through its life cycle easily and without facing failure. This is a prerequisite for being agile, which needs a system to be able to change rapidly [19].

To understand this better, consider a modular die, it may be easy to change a module, but it could be time-consuming as it may need a production line to be shut down, and perhaps it takes a lot of time to restart it. Though, in an agile system, a die should be able to quickly be updated on the production line even if it requires not easy-to-use tools to do this task. In the same way, robustness is accomplished when the system is incentive toward its environment, and

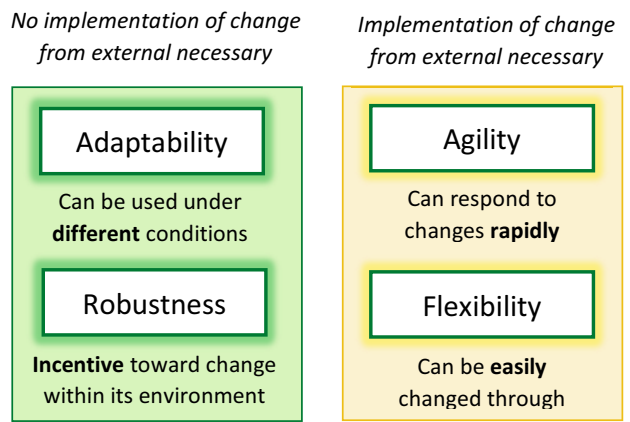

Figure 1. Various aspects of changeability it is a prerequisite for being adaptable which is when the system can be used under different conditions. For example, a component's tolerances in the assembly line could be robust toward small manufacturing deficiencies and still be assembled. Yet, we can say it is adaptable when this component is able to be used for a totally different product than the one it is initially developed for. This is a particularly useful attribute in product platforms when we cannot foresee the future variants of a product family. Lacking any of these attributes, in a system like product realization, can cause failure directly or indirectly by propagation of change to other phases.

Ross et al. [14] also made an attempt to define changeability in terms of several system characteristics, namely, Robustness, Flexibility, Adaptability, Scalability, and Modifiability. In this way, first, they presented an enhanced definition of change in terms of the transition from one state to another. This definition characterizes a change based on three elements: agent of a change, mechanism, and its effect. These elements are shown in Figure 2 (a). The change is represented by a path from one state to another and changeability is defined as how easy a system could complete this path. The agent is a force that drives the change and it could be triggered supervised, like a decision (e.g. mechanical mechanism, software, etc.) or unsupervised like a natural force (e.g. Gravity, wind, etc.). The agent's location is another attribute that is most useful in making a distinction between flexibility and adaptability, if it is an internal agent, the system could be named as adaptable. If the agent is external to the system, the system is called flexible [20]. This concept is also depicted in Figure 2 (b). In this definition, the distinction between flexibility and adaptability also depends on the type of change. System boundary plays an important role in removing the ambiguity in this distinction. The change effect is another element that is defined as the difference between the initial and late state of the system. This element helps to make distinction between modifiability, scalability, and robustness.

To illustrate better, consider a system that has three parameters: $a, b$, and c. And each of these parameters could have three values, which are labeled sets. $a=\left[a_{1}, a_{2}, a_{3}\right]$ and $b$ $=\left[b_{1}, b_{2}, b_{3}\right]$ and $c=\left[c_{1}, c_{2}, c_{3}\right]$. In this way, system $X$ could be defined as $X=\left(a_{1}, b_{2}, c_{3}\right)$. Suppose designers change the environment that this system is currently working. If $X$ maintains its performance, then we call it a robust system. If designers change one of the parameters of the $X$ so it is equal as $X=\left(\mathrm{a}_{1}, \mathrm{~b}_{2}, \mathrm{c}_{2}\right)$ and the system runs without any 
performance loss then we call this system scalable. Finally, if the designer introduces a new set, as d, and changes the system's parameters to be $X=\left(\mathrm{a}_{1}, \mathrm{~b}_{2}, \mathrm{c}_{4}, \mathrm{~d}\right)$ and still the system is functioning then the system is modifiable. These characteristics are shown in Figure 2 (c). The last element is the change mechanism which is defined with the path that is used by the system to get to a later state. Depending on the mechanism, system cost and revenue may change. Figure 2 (c) shows 4 different change mechanisms with different costs between the same initial and later states.

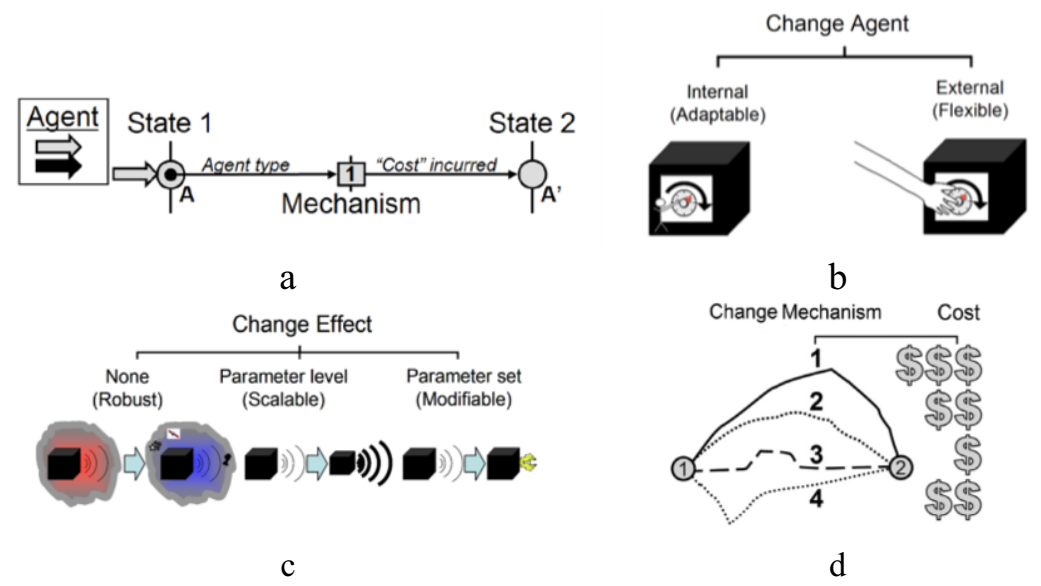

Figure 2. Changeability elements and distinction they make between ilities [14].

De Weck, et al. in the article [21] investigated relationships and semantic sets amongst system lifecycle properties, and they basically give the same definitions and classifications as presented by [14]. Yet in their book which has published one year ahead [22] they devoted a chapter on defining life cycle properties of engineering systems and presented some other definitions. Other students from MIT system engineering advance research initiatives that have published definitions in this area have identical definitions such as [23] and [24].

Uncertainties are defined by [25] as "the inability to determine the true state of affairs of a system" or "things that are unknown or known only imprecisely" by [26]. These undesirable effects are sometimes the reason behind a late decision or change in the systems that end up propagating in the systems or more specifically in the product realization realm. Some authors acknowledge this fact by focusing on characteristics that can make a system incentive toward uncertainties [27]. They compare several system characteristics (ilities) that act as a protection against uncertainties and attempts to mitigate them without removing their source. The paper [27] employs several perspectives on reliability. And based on these three viewpoints the article defines reliability as the ability of a system to perform as expected in a stable environment. In this way, reliability is used as an umbrella concept for several other characteristics. Robustness is defined as the ability of a system, as-built/designed, to do its basic job in uncertain or changing environments. This attribute is considered passive, meaning it is designed to ensure its capabilities and to withstand the uncertainties. Adaptability is defined as the ability of a system to be modified in order to do its basic job in uncertain or changing environments, changing requirements are omitted from this definition. Adaptability is considered active with variable structure meaning the system changes 
itself to adapt to the new environment. For example, some of the high-tech car spoilers adjust their height to compensate for the vehicle's aerodynamic shape at varying speeds. In Robustness the change is intrinsic, and the protection is passive. This difference is illustrated in Table 2 for different system properties [27]. Versatility is defined as the ability of a system, as-built/designed, to do jobs not originally included in its requirements. And resilience in a narrow sense focuses on a system's recovery from perturbation. Flexibility is defined as the ability of a system to be modified to do its basic job or jobs not originally included in the definition of the system's requirements in uncertain or changing environments. Flexibility considered active protection and also it was argued that it could work as a facilitator for robustness.

Table 2. classification of conceptual approaches to system protection against uncertainty.

\begin{tabular}{cccccc}
\hline Concept & $\begin{array}{c}\text { Variable } \\
\text { Requirement }\end{array}$ & $\begin{array}{c}\text { Variable } \\
\text { Structure }\end{array}$ & $\begin{array}{c}\text { Variable } \\
\text { Environment }\end{array}$ & $\begin{array}{c}\text { Active } \\
\text { considered }\end{array}$ & Source of Uncertainty \\
\hline Reliability & & & & & System \\
\hline Robustness & & & $\sqrt{ }$ & $\sqrt{ }$ & System + Environment \\
\hline Adaptability & & $\sqrt{ }$ & $\sqrt{ }$ & & System + Environment \\
\hline Versatility & $\sqrt{ }$ & & & $\sqrt{ }$ & System + Environment+ Requirement \\
\hline Resilience & $\sqrt{ }$ & & $\sqrt{ }$ & $\sqrt{ }$ & System + Environment+ Requirement \\
\hline Flexibility & $\sqrt{ }$ & $\sqrt{ }$ & $\sqrt{ }$ & & \\
\hline
\end{tabular}

In another research [28] has introduced an ontological framework to clarify different aspects of flexibility, adaptability, robustness. They prepared three different tables, each for one of the attributes, namely, flexibility, robustness, and adaptability. Based on the proposed framework the authors attempted to suggest a more clarifying definition on mentioned ilities. Flexibility was summed up as the measure of how easily a system's capabilities can be modified in response to external change. Adaptability was concluded to be the measure of how effectively a system can modify its own capabilities in response to change after it has been fielded. Finally, robustness regarded as the measure of how effectively a system can maintain a given set of capabilities in response to external changes after it has been fielded. In this sense, a robust system handles the change in the environment without really changing itself.

\section{Comparison and analysis}

As discussed in the previous section, some of the system properties (ilities) can be defined in association with addressing the changes and butterfly effects. Studying the change in generic form enables a transdisciplinary approach that can fit well in various disciplines of product realization. Whether it is a comprehensive manufacturing system or a system for the detailed design, it can still fit in discussed definitions. Therefore, running a systemlevel research clarification phase at the beginning of the this research project was essential. Table 3 summarizes the results with the aim of giving a brief overview and a comparison between ontological studies at hand.

The citation of each article was also brought in the table 2 and it is basically an indication of the impact of the proposed definition. As can be seen, Flexibility, Adaptability, and Robustness are the most repeated system properties between different papers in the product domain. For the production domain, Flexibility and Reconfigurability are the most repeated ones. The difference between the two domains

\footnotetext{
${ }^{2}$ Pulled out at 30 January 2020 at the time of writing this article.
} 
shows that changes can mean different things in two domains. For transdisciplinary nature of the Butterfly Effect project, meaning that it aims to study change propagations between several disciplines, this is very important. Repetition of ilities used in defining changeability in each author is also important since it shows unanimous definition and agreement of authors for defining and classifying these terms. There are some other attempts like [30] and [31] to classify these definitions in different structures but since they don't give any definition of their own, their articles are not included in this table. Also, a problem with their approach is that it is hard to compare different views on various definitions.

Table 3. Different ilities each author used for their definition.

\begin{tabular}{clcl}
\hline Domain & \multicolumn{1}{c}{ Article } & Times Cited & \multicolumn{1}{c}{ Used ilities } \\
& Fricke and Schulz [19] & 417 & $\begin{array}{l}\text { Changeability (Flexibility, Adaptability, Agility, } \\
\text { Robustness) }\end{array}$ \\
\cline { 2 - 4 } & Ross, et al. [14] & 351 & $\begin{array}{l}\text { Changeability (Robustness, Modifiability, Scalability) also } \\
\text { Changeability (Flexibility, Adaptability) }\end{array}$ \\
\cline { 2 - 4 } & 279 & $\begin{array}{l}\text { Flexibility, Adaptability, Agility, Evolvability, } \\
\text { Reconfigurability, Scalability, Extensibility }\end{array}$ \\
\cline { 2 - 4 } & DeWeck, et al. [22] & 49 & $\begin{array}{l}\text { Reliability (Flexibility, Adaptability, Robustness, } \\
\text { Versatility, Resilience) }\end{array}$ \\
\cline { 2 - 4 } Chalupnik, et al. [27] & 65 & $\begin{array}{l}\text { (Flexibility, Adaptability, Robustness), and } \\
\text { Agility, Changeability, Versatility }\end{array}$ \\
\hline Ryan, et al. [28] & 964 & $\begin{array}{l}\text { Changeability (Changeover-ability, Reconfigurability, } \\
\text { Flexibility, Transformability, Agility) }\end{array}$ \\
\hline Terkaj, et al. [29] & 73 & $\begin{array}{l}\text { Flexibility (Flexibility, Reconfigurability, Changeability) } \\
\text { also (Range, Resolution, Mobility, Uniformity) }\end{array}$ \\
\hline Benkamoun [17] & 6 & $\begin{array}{l}\text { Changeability [Flexibility (Built-in, Changeover-ability), } \\
\text { Reconfigurability (convertibility, extensibility)] }\end{array}$ \\
\hline
\end{tabular}

To avoid disperse definitions in several tables and better illustrate and compare definitions given by each author on the mentioned terms, Table 4 is put together in this study. This Table summarizes papers in the product domain with a gist of each term's definition. The numbers in the table refer to the definitions listed beside the table. For example, Fricke and Schulz for Flexibility used definitions 1 and 7 (or at least these numbered definitions are aligned with the author's discussion). An interesting inference is that the definition for adaptability and robustness is almost unanimous by different authors but there is no consensus about the definition of flexibility. For example, the definition "Maintaining capabilities in changing environment" is more or less repeated by many authors for robustness and the same is true for the definition of "System can change itself actively in response to varying condition" for adaptability. One can conclude that the authors in system engineering believe adaptability to be the intrinsic ability of a system to change itself and adapt to changes. As for the robustness, the agreed-upon definition is the ability to maintain its capabilities in a changing environment. It is only Ross, et al. [14] that doesn't approve this definition for adaptability.

However, for flexibility, different authors give different definitions and no unanimity can be seen between them. Interestingly, flexibility is the only attribute that is repeated and shared between the product and the production domain (base on table 3 ). It cannot be determined if this is the reason for such variations in the definition of flexibility. Nevertheless, ambiguity in this filed can be troublesome for scholars and damage the development of the field. To fully understand the trend in literature and the Internet about these three ilities (Flexibility, Adaptability, and Robustness), a comparison was made which is brought in Figure 3. This comparison was previously done in 2011 by De Weck et al. [22] and we used their data to find out what has changed in this last decade. 
Table 4. Definitions that different authors used and their overlap.

1 - Being able to change easily (effort) in a passive way

\begin{tabular}{|c|c|c|c|c|}
\hline 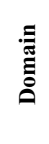 & Article & 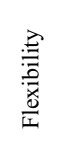 & 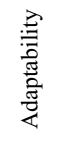 & 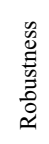 \\
\hline \multirow{5}{*}{ } & Fricke and Schulz [19] & 1,7 & 2,8 & 3,8 \\
\hline & Ross, et al. [14] & 4 & 5 & - \\
\hline & DeWeck, et al. [22] & 6,1 & 2,4 & 3,9 \\
\hline & Chalupnik, et al. [27] & 10 & 2 & 3 \\
\hline & Ryan, et al. [28] & 1,4 & 2 & 3,4 \\
\hline
\end{tabular}

2- The system can change itself actively in response to the varying condition

3- Maintaining capabilities in changing environment

4- Force instigator (agent) for the change is external to the system

5- Change in response to an internal agent

6- Umbrella term for several other ilities

7- Changes from external must be implemented to cope with changing environments.

8- No implementation of change from external is necessary

9- Sub-ility for the Resilience umbrella

10- Ability to be modified to do jobs not originally intended by the original design

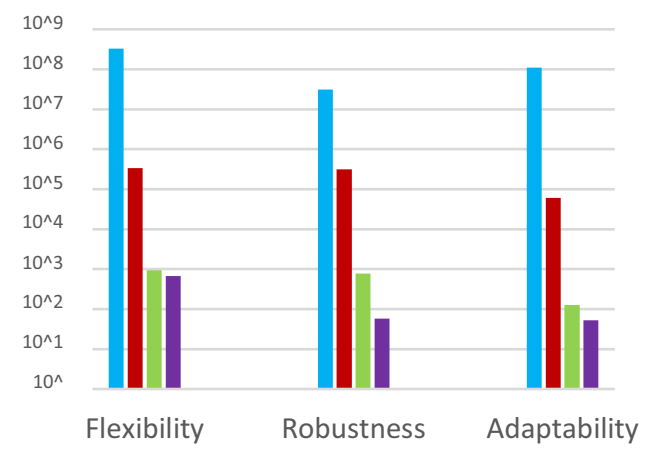

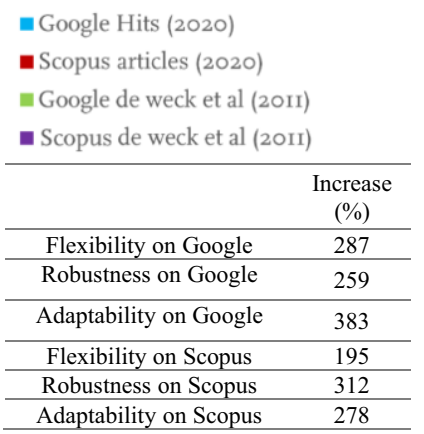

Figure 3: Trend in three different system properties over the past decade.

In this figure, numbers from [22] are compared to numbers that were extracted by this paper. What is interesting is the amount of increase in these numbers. For example, the bars over robustness, shows that the number of articles increased $312 \%$ in Scopus (purple bar to red) from 2011 to 2020. Yet this increase for flexibility in Scopus is $195 \%$, which is very low comparing to robustness growth. The fact robustness in Scopus has experienced more increase in comparison to flexibility, shows although flexibility was well published in previous years, Robustness is getting much more attention nowadays from scholars. This is interesting from the Butterfly Effect research project's point of view to know which system properties are trending in academic literature. Another interesting fact is adaptability hits on Google which has been increased by $383 \%$ from 2011 to 2020, still, this increase is Scopus is $278 \%$. This also shows that adaptability is not getting the recognition it used to get from scholars and academic society. Overall, one can predict that robustness as a system property is on the verge of outburst and we can expect to see more papers on this system property in the years to come. This also helps us to focus more on Robustness in future works as a trend in literature. 


\section{Conclusion}

Changes are inevitable through every phase of product realization. They may cause a problem for the system or just lead to the other changes, propagating through different phases of product realization. Most used change-related ilities are extracted as Flexibility, Adaptability, and Robustness. A consensus definition was identified for each of these terms. The performed historical comparison shows that robustness is getting more and more recognition from scholars and it is indeed a trend in the future literature. Results show that three mentioned system properties can be used to address the change propagation in the field of product realization. The next step in the Butterfly Effect research project will be to look at each of these ilities individually to see what supports have been developed to address each of them. The final goal is to develop sophisticated support for change propagations. We will also run interviews with the companies to find case studies to evaluate our proposed support.

\section{Acknowledgment}

This work has been carried out within the project Butterfly Effect in the school of engineering, Jönköping University. The authors would like to acknowledge three companies involved in the research projects and also Swedish Knowledge Foundation ('KK-Stiftelsen') for the finacial support.

\section{References}

[1] H.-P. Wiendahl and C. Heger, Justifying changeability. A methodical approach to achieving cost effectiveness, Journal for Manufacturing science and Production, 2004, Vol. 6, No. 1-2, pp. 3340 .

[2] N. Benkamoun, Systemic design methodology for changeable manufacturing systems, PhD thesis, University Blaise Pascal - Clermont-Ferrand II, NNT : 2016CLF22723, 2016.

[3] E. Lorenz, The butterfly effect, World Scientific Series on Nonlinear Science Series A, 2000, Vol. 39, pp. 91-94.

[4] G. Li, S. H. Yu, and W. P. He, Research on Lorentz Chaos in Complex Product Family Shape, (in English), 2017 2nd Ieee International Conference on Wireless Communications, Signal Processing and Networking (Wispnet) , 2017, pp. 856-859.

[5] I. Nonaka and H. Takeuchi, The knowledge-creating company: How Japanese companies create the dynamics of innovation. Oxford university press, 1995.

[6] C. Eckert, P. J. Clarkson, and W. Zanker, Change and customisation in complex engineering domains, (in English), Research in Engineering Design, Mar 2004, Vol. 15, No. 1, pp. 1-21. R. Stolt, J. Johansson, S. André, T. Heikkinen, and F. Elgh, How to Challenge Fluctuating Requirements: Results from Three Companies, Advances in Transdisciplinary Engineering, Vol. 3, 2016, pp. 1061-1070.

[8] T. Heikkinen, J. Johansson, and F. Elgh, Assessment of Simulation Ready CAD Models in a SetBased Concurrent Engineering Context, in DS 84: Proceedings of the DESIGN 2016 14th International Design Conference, 2016, pp. 649-658.

[9] J. Johansson, Mastering the Butterfly Effects within Knowledge Intensive Product Realization, School of Engineering, Jönköping University, 2019, pp. 1-64.

[10] A. M. Ross and D. H. Rhodes, Towards a prescriptive semantic basis for change-type ilities, Procedia Computer Science, 2015, Vol. 44, pp. 443-453.

[11] D. K. Hitchins, Systems engineering: a 21st century systems methodology. John Wiley \& Sons, Hoboken, 2008.

[12] N.N., ESD Symposium Comittee Overview: Engineering Systems Research and Practice, E.S. Committee, 2002.

[13] A. P. Schulz and E. Fricke, Incorporating flexibility, agility, robustness, and adaptability within the design of integrated systems-key to success?, in Gateway to the New Millennium. 18th Digital 
Avionics Systems Conference. Proceedings (Cat. No. 99CH37033), 1999, vol. 1, pp. 1. A. 2-1. A. 2: IEEE.

[14] A. M. Ross, D. H. Rhodes, and D. E. Hastings, Defining changeability: Reconciling flexibility, adaptability, scalability, modifiability, and robustness for maintaining system lifecycle value, Systems Engineering, 2008, Vol. 11, No. 3, pp. 246-262.

[15] A. M. Ross and M. SE, Contributing toward a Prescriptive "Theory of Ilities", 1st Annual SERC Technical Review. RT-113 Foundations, 2014.

[16] H.-P. Wiendahl et al., Changeable manufacturing-classification, design and operation, CIRP annals, 2007, Vol. 56, no. 2, pp. 783-809.

[17] N. Benkamoun, An Intelligent Design Environment for Changeability Management - Application to Manufacturing Systems, (in English), Ds 80-3 Proceedings of the 20th International Conference on Engineering Design (Iced 15) Vol 3: Organisation and Management, 2015.

[18] K. B. Clark and T. Fujimoto, Product development performance: Strategy, organization, and management in the world auto industry, Harvard Business School Press, Boston, 1991.

[19] E. Fricke and A. P. Schulz, Design for changeability (DfC): Principles to enable changes in systems throughout their entire lifecycle, Systems Engineering, 2005, vol. 8, no. 4, https://doi.org/10.1002/sys.20039.

[20] A. M. Ross, Managing unarticulated value: changeability in multi-attribute tradespace exploration, $\mathrm{PhD}$ thesis, MIT, Boston, 2006.

[21] O. L. De Weck, A. M. Ross, and D. H. Rhodes, Investigating relationships and semantic sets amongst system lifecycle properties (ilities), ESD Working Papers;ESD-WP-2012-12, MIT, Boston, 2012.

[22] O. L. DeWeck, D. Roos, and C. L. Magee, Engineering Systems: Meeting Human Needs in a Complex Technological World, MIT Press, Boston, 2011.

[23] J. C. Beesemyer, Empirically characterizing evolvability and changeability in engineering systems, MSc thesis, MIT, Boston, 2012.

[24] M. E. Fitzgerald, Managing uncertainty in systems with a valuation approach for strategic changeability, MSc thesis, MIT, Boston, 2012.

[25] Y. Y. Haimes, Risk modeling, assessment, and management. John Wiley \& Sons, Hoboken, 2005.

[26] H. McManus and D. Hastings, 3.4. 1 A framework for understanding uncertainty and its mitigation and exploitation in complex systems, in INCOSE international symposium, 2005, vol. 15, no. 1, pp. 484-503.

[27] M. J. Chalupnik, D. C. Wynn, and P. J. Clarkson, Comparison of ilities for protection against uncertainty in system design, Journal of Engineering Design, 2013, Vol. 24, No. 12, pp. 814-829. E. T. Ryan, D. R. Jacques, and J. M. Colombi, An ontological framework for clarifying flexibilityrelated terminology via literature survey, Systems Engineering, 2013, Vol. 16, No. 1, pp. 99-110.

[29] W. Terkaj, T. Tolio, and A. Valente, Focused flexibility in production systems, in H. ElMaraghy (ed.) Changeable and reconfigurable manufacturing systems: Springer, London, 2009, pp. 47-66.

[30] E. F. Colombo, G. Cascini, and O. L. de Weck, Classification of Change-Related Ilities Based on a Literature Review of Engineering Changes, Journal of Integrated Design \& Process Science, 2016, Vol. 20, no. 4, pp. 3-23.

[31] B. P. Sullivan, M. Rossi, and S. Terzi, A Review of Changeability in Complex Engineering Systems, IFAC-PapersOnLine, 2018, Vol. 51, no. 11, pp. 1567-1572. 